\title{
Optical Control of Topological Quantum Transport in Semiconductors
}

\author{
Wang Yao* A. H. MacDonald, and Qian Niu \\ Department of Physics, The University of Texas, Austin, Texas 78712
}

(Dated: August 15, 2018)

\begin{abstract}
Intense coherent laser radiation red-detuned from absorption edge can reactively activate sizable Hall type charge and spin transport in n-doped paramagnetic semiconductors as a consequence of $k$-space Berry curvature transferred from valence band to photon-dressed conduction band. In the presence of disorder, the optically induced Hall conductance can change sign with laser intensity.
\end{abstract}

PACS numbers: 73.43.-f, 72.25.Dc, 78.20.Ls, 78.20.Jq

Modification of material properties by external fields is of fundamental interests in condensed matter physics. Well known examples include optical Kerr nonlinearity, magneto-resistance, and the Hall effect. These phenomena enable externally controllable devices with importance in technologies. Semiconductors offer an ideal platform to explore control possibilities due to rich behaviors under magnetic, electric, and optical perturbations. In particular, with advances in laser technology, optically activated novel properties in semiconductors can now be explored with intense coherent lasers [1] and are motivated by the need for ultra-fast switchable devices.

In this letter, we predict coherently photoinduced Hall type charge and spin transport in n-doped paramagnetic semiconductors in which conduction band spin-orbit coupling can be absent. In semiconductors, an optical field couples the lowest $s$-like conduction band and the top $p$-like valance bands with a sizable interband matrix element. When the light is far red-detuned in frequency from the absorption edge, the influence on the electronic system is reactive, rather than dissipative as in most previous photoinduced transport schemes [2, 3, 4]. The off-resonance coherent laser beam renormalizes the band structure [5], in a fashion analogous to the dynamical Stark effect in atomic physics. $k$-space Berry curvatures which originate from strong spin-orbit coupling in the valance bands are redistributed in the photon-dressed bands and the occupied electronic states acquires nontrivial topological properties [6, 7]. Circularly polarized light breaks time reversal symmetry and leads to an anomalous Hall effect (AHE) [8, 9, 10, 11, 12, 13, 14]. With linearly polarized light, time reversal symmetry forbids a net charge Hall current, but a DC electric field can drive a pseudo-spin current in the perpendicular direction, i.e. bidirectional Hall flow of the dressed electrons similar to the spin Hall effect (SHE) [15, 16, 17]. The population proportion of the two species of electrons is driven into imbalance at the edges of the light irradiation area, which results in long-lived pure spin accumulations of conduction band electrons when the light is turned off adiabatically.

The AHE has been one of the most fundamental yet controversial issues in condensed matter physics. Despite its extensive use for sample characterization in ferromag- netic systems, there have long been debates if the AHE originates entirely from spin-dependent scattering at impurities, or has important topological contribution from $k$-space Berry curvature [8, 9, 10, 11, 12, 13, 14]. The SHE offers a promising avenue towards semiconductor spintronics based on pure spin transport, yet the definition of spin current remains a controversial issue in strong spin-orbit coupled systems [18]. Not coincidentally, it has been a challenge to relate the spin current to the experimentally observable spin accumulations at sample edges, since it has been realized that the latter can also be induced locally and is dependent on boundary conditions.

As an attempt to contribute to these conceptual debates that are critical for making the connection between experimental and theoretical studies, we choose to illustrate our idea by applying it to an n-doped quantum well (QW) of III-V compounds where structure inversion asymmetry (SIA) induced spin-orbit coupling is absent [19]. We predict that the AHE and SHE can be activated/deactivated in this paramagnetic system by switching on/off the light field only, which can be a clear demonstration of the topological contributions. This system has the unique feature that it consists of two decoupled subspaces related by a time-reversal operation [20], which unambiguously leads to the definition of the pseudo-spin current as the only source of the spin accumulations with out-of-plane polarization at the light irradiation edges.

We first explain the band structure renormalization by light for noninteracting electrons and then consider the effect of interactions. The explicit time dependence in the coupling with a coherent laser field of frequency $\omega_{0}$ is removed by a canonical transform $U(t)=$ $\exp \left(-\mathrm{i} \omega_{0} \mathrm{t} \sum_{\mathbf{k}} \mathrm{a}_{\mathrm{c}, \mathbf{k}}^{\dagger} \mathrm{a}_{\mathbf{c}, \mathbf{k}}\right)$, so that in this rotating frame $(\mathrm{RF})$, the Hamiltonian reads [5]

$$
\begin{aligned}
H_{\mathrm{RF}} & =\sum_{\mathbf{k}}\left(\varepsilon_{c}(\mathbf{k})-\omega_{0}\right) a_{c, \mathbf{k}}^{\dagger} a_{c, \mathbf{k}}+\sum_{\mathbf{k}, j} \varepsilon_{v_{j}}(\mathbf{k}) a_{v_{j}, \mathbf{k}}^{\dagger} a_{v_{j}, \mathbf{k}} \\
& +\sum_{\sigma} \sum_{\mathbf{k}, j} \Omega_{\sigma, j}(\mathbf{k}) a_{c, \mathbf{k}}^{\dagger} a_{v_{j}, \mathbf{k}}+\text { c.c. }
\end{aligned}
$$

where $a_{c, \mathbf{k}}^{\dagger}$ and $a_{v_{j}, \mathbf{k}}^{\dagger}$ are the particle creation operators in the conduction band and the $j$ th valance band. $\sigma$ 
denotes the polarization components of the light, and the Rabi frequency $\Omega_{\sigma, j}$ is proportional to the laser amplitude and the interband optical matrix element. Eq.(11) is diagonalized by a linear transformation, $H_{\mathrm{RF}}=$ $\sum_{\mathbf{k}, m} \tilde{\varepsilon}_{m}(\mathbf{k}) \tilde{a}_{m, \mathbf{k}}^{\dagger} \tilde{a}_{m, \mathbf{k}}$, where $\tilde{a}_{m, \mathbf{k}}$ denotes the creation of a particle in the photon-dressed band with the new dispersion $\tilde{\varepsilon}_{m}(\mathbf{k})$. We still use the subscript $c$ to denote the dressed band with highest energy in the RF, $\tilde{a}_{c, \mathbf{k}} \equiv \alpha(\mathbf{k}) a_{c, \mathbf{k}}+\sum_{j} \beta_{j}(\mathbf{k}) a_{v_{j}, \mathbf{k}}$.

In absence of the laser field, the n-doped system has a filled $k$-space region $A$ in the conduction band. It is convenient to use a hole picture in which the above configuration reads $|G\rangle=\prod_{\mathbf{k} \notin A} b_{c,-\mathbf{k}}^{\dagger}\left|\Phi_{0}\right\rangle .\left|\Phi_{0}\right\rangle$ denotes complete filling of all bands, and $b_{c,-\mathbf{k}}^{\dagger} \equiv a_{c, \mathbf{k}}$ is hole creation operator for unfilled states. Without conduction band spin-orbit coupling, the states $b_{c,-\mathbf{k}}^{\dagger}\left|\Phi_{0}\right\rangle$ have zero Berry curvatures and hence $|G\rangle$ has no topological contribution to the Hall conductance. If the laser field, red-detuned from the absorption edge $\omega_{\text {edge }} \equiv \min _{\mathbf{k} \notin A}\left(\varepsilon_{c}(\mathbf{k})-\varepsilon_{v_{j}}(\mathbf{k})\right)$, is adiabatically switched on, the system will evolve from $|G\rangle$ to the dressed state $|\tilde{G}\rangle=\prod_{\mathbf{k} \notin \tilde{A}} \tilde{b}_{c,-\mathbf{k}}^{\dagger}\left|\Phi_{0}\right\rangle$ where $\tilde{b}_{c,-\mathbf{k}}^{\dagger} \equiv \tilde{a}_{c, \mathbf{k}}$. We have assumed elastic scattering by impurities and temperatures low enough $(<100 \mathrm{~K})$ so that the phonon bath cannot supply the energy to excite the electron out of the lower dressed bands. Weak spontaneous emission of photons by the virtual excitations is also neglected for the moment. In the cases considered, the new equilibrium $k$-space filling $\tilde{A}$ (the lowest energy configuration in the RF) differs only slightly from $A$. Because of the admixture of the valance bands which have strong spin-orbit coupling, the dressed band $\tilde{b}_{c, \mathbf{k}}^{\dagger}\left|\Phi_{0}\right\rangle$ has non-zero Berry curvature $\mathbf{F}^{c}(\mathbf{k})=\nabla_{\mathbf{k}} \times \mathbf{R}^{c}(\mathbf{k})$ where $\mathbf{R}^{c} \equiv i\left\langle\Phi_{0}\left|\tilde{b}_{c, \mathbf{k}} e^{i \mathbf{k} \cdot \mathbf{r}}(\partial / \partial \mathbf{k}) e^{-i \mathbf{k} \cdot \mathbf{r}} \tilde{b}_{c, \mathbf{k}}^{\dagger}\right| \Phi_{0}\right\rangle$ [7]. The dressed state $|\tilde{G}\rangle$ thus acquires topological transport properties.

In a direct bandgap III-V semiconductor, the electronic states affected by light are those near the $\Gamma$ point. It is a good approximation to neglect interband scattering and interband exchange terms in Coulomb interaction since the periodic part of Bloch functions are orthogonal between different bands near the $\Gamma$ point [5]. For the same reason, we also assume no interband scattering by phonons and smooth disorder. Thus, in the RF, the Coulomb interactions and the coupling to phonon and impurity potentials has the time-independent form.

Coulomb interactions which have several major effects are well accounted for in optically excited semiconductors by the Hartree Fock approximation [5]. First, the band dispersion $\varepsilon_{l}(\mathbf{k})$ includes the interaction energies between the occupied states in the ground state configuration $|G\rangle$. Second, considering the interaction between the virtual excitations [5], the transitions energies are further renormalized, $\varepsilon_{c}(\mathbf{k}) \rightarrow \varepsilon_{c}(\mathbf{k})-\sum_{j, \mathbf{k}^{\prime} \notin \tilde{A}} V\left(\mathbf{k}^{\prime}-\mathbf{k}\right)\left|\beta_{j}\left(\mathbf{k}^{\prime}\right)\right|^{2}$ and $\varepsilon_{v_{j}}(\mathbf{k}) \rightarrow \varepsilon_{v_{j}}(\mathbf{k})+\sum_{\mathbf{k}^{\prime} \notin \tilde{A}} V\left(\mathbf{k}^{\prime}-\mathbf{k}\right)\left|\beta_{j}\left(\mathbf{k}^{\prime}\right)\right|^{2}$, where $V\left(\mathbf{k}^{\prime}-\mathbf{k}\right)$ is the screened Coulomb interaction. The effective Rabi frequency is also renormalized $\Omega_{\sigma, v_{j}}(\mathbf{k}) \rightarrow$
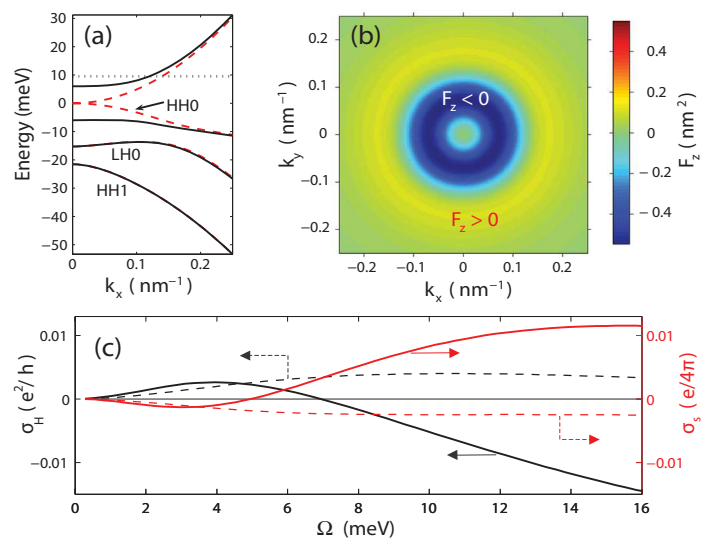

FIG. 1: (Color online) (a) Dispersion relations of the dressed bands (solid curves) in the $\mathcal{H}_{-}$subspace with a $\sigma+$ polarized laser $\left(\Omega \equiv\left|\Omega_{+, \mathrm{HH}}\right|=6 \mathrm{meV}\right)$. Dashed curves denotes the undressed bands in the rotating frame. The horizontal dotted line denote the Fermi energy. (b) The corresponding Berry Curvature distribution of the top dressed band $\tilde{b}_{c, \mathbf{k}}^{\dagger}\left|\Phi_{0}\right\rangle$. (c) Charge Hall conductance $\sigma_{\mathrm{H}}$ (black curves) and pseudospin Hall conductance $\sigma_{s}$ (red curves) as a function of laser strength $\Omega$. Dashed curves denote the clean contribution and solid curves include disorder effects. $k_{B} T=0.5 \mathrm{meV}, \omega_{0}=$ $E_{g}$, and the electron density is fixed at $n=9 \times 10^{12} \mathrm{~cm}^{-2}$.

$\Omega_{\sigma, v_{j}}(\mathbf{k})+\sum_{\mathbf{k}^{\prime} \notin \tilde{A}} V\left(\mathbf{k}^{\prime}-\mathbf{k}\right) \alpha\left(\mathbf{k}^{\prime}\right) \beta_{j}^{*}\left(\mathbf{k}^{\prime}\right)$. The diagonalization of Eq.(11) has to be performed self-consistently. We also note that the optical absorption edge becomes $\omega_{\text {edge }} \equiv \min _{\mathbf{k} \notin A}\left(\varepsilon_{c}(\mathbf{k})-\varepsilon_{v_{j}}(\mathbf{k})-\epsilon_{b}\right)$ where $\epsilon_{b}$ is the binding energy to form an exciton.

We consider an n-doped GaAs/AlGaAs QW, with symmetric confinement in the growth $(z)$ direction. For the conduction band electrons, Rashba spin-orbit coupling vanishes since there is no SIA, and we neglect the weak Dresselhaus spin-orbit coupling except for the consequence of a finite spin relaxation time in the presence of phonon scattering [21]. QW confinement splits the $\Gamma_{6}$ $(S=1 / 2)$ bulk conduction band into subbands denoted as $\left|S_{z}, \mathbf{k}_{\perp}, l_{c}\right\rangle$ where $S_{z}= \pm 1 / 2$ is the spin $z$ quantum number, $\mathbf{k}_{\perp}$ the in-plane wavevector, and $l$ the quantum number of the QW confinement. The $\Gamma_{8}(J=3 / 2)$ valance bands in bulk material is described by the standard Luttinger Hamiltonian [22]. The eigenstates of $\hat{J}_{z}$ together with the QW confinement define a basis sets: $\left|J_{z}, \mathbf{k}_{\perp}, l_{v}\right\rangle$, similar to that of the conduction subbands. Off-diagonal terms in the Luttinger Hamiltonian are the origin of $k$-space Berry curvature, and they couple the valance subbands at the same $\mathbf{k}_{\perp}$ with the selection rule: (i) $\Delta J_{z}= \pm 1$ and $\Delta l_{v}$ odd (a change of parity in $z$ direction); or (ii) $\Delta J_{z}= \pm 2$ and $\Delta l_{v}$ even (no parity change). A light field with $\sigma$ polarization propagating in the $z$ direction couples the conduction and valance subbands with the selection rules: $S_{z}=J_{z}+\sigma$ and $l_{c}-l_{v}$ even. Therefore, we always have two decoupled subspaces: (i) $\mathcal{H}_{+}=\left\{\left|S_{z}=1 / 2, l_{c}=e\right\rangle,\left|S_{z}=-1 / 2, l_{c}=o\right\rangle, \mid J_{z}=\right.$ 

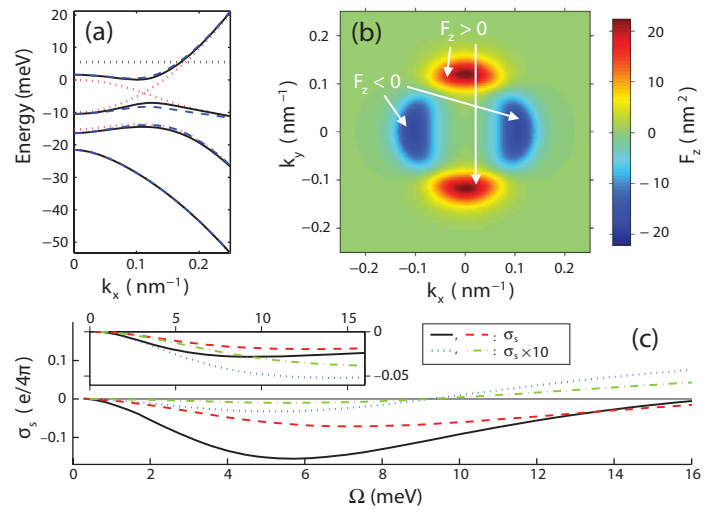

FIG. 2: (Color online) (a) Dispersion relations of the dressed bands in subspace $\mathcal{H}_{-}$with a $X$ polarized laser $\left(\omega_{0}-E_{g}=10\right.$ $\mathrm{meV}$ and $\Omega=6 \mathrm{meV}$ ). Solid (dashed) curves denote the band dispersions along $k_{x}=0\left(k_{y}=0\right)$. The Red dotted curves denotes the undressed bands in the RF. The horizontal dotted line denote the Fermi energy $\left(n=18 \times 10^{12} \mathrm{~cm}^{-2}\right)$. (b) The corresponding Berry Curvature distribution of the top dressed band $\tilde{b}_{c, \mathbf{k}}^{\dagger}\left|\Phi_{0}\right\rangle$. (c) Pseudo-spin Hall conductance as a function of laser Rabi frequency $\Omega$. The inset shows the clean contribution. The parameters are, respectively, solid curve: $\omega_{0}-E_{g}=13 \mathrm{meV}$ and $n=18 \times 10^{12} \mathrm{~cm}^{-2}$; dashed: $\omega_{0}-E_{g}=10 \mathrm{meV}$ and $n=18 \times 10^{12} \mathrm{~cm}^{-2} ;$ dotted: $\omega_{0}=E_{g}$ and $n=9 \times 10^{12} \mathrm{~cm}^{-2}$; dash-dotted: $\omega_{0}-E_{g}=-4 \mathrm{meV}$, and $n=9 \times 10^{12} \mathrm{~cm}^{-2}$. The latter two curves are horizontally scaled by a factor of $10 . k_{B} T=0.5 \mathrm{meV}$.

$\left.3 / 2, l_{v}=e\right\rangle,\left|J_{z}=-1 / 2, l_{v}=e\right\rangle, \mid J_{z}=-3 / 2, l_{v}=$ $\left.o\rangle,\left|J_{z}=1 / 2, l_{v}=o\right\rangle\right\}$ where we have used $e(o)$ to denote even (odd) integer; (ii) $\mathcal{H}_{-}$is the time reversal of $\mathcal{H}_{+}$. For typical QW, the inclusion of lowest conduction subbands with $l_{c}=0$, light-hole subbands with $l_{v}=0$, and heavy-hole subbands with $l_{v}=0,1$ suffices since all other subbands are faraway in energy.

Numerical evaluations were performed for a QW with thickness $d=10 \mathrm{~nm}$. For the QW layer and the barrier, we assume the same Luttinger parameters $\left(\gamma_{1}=6.85\right.$, $\gamma_{2}=2.1$, and $\left.\gamma_{3}=2.9\right)$ and conduction band effective mass $\left(m_{c}=0.078 m_{e}\right)$. We simply assume $x, y$, and $z$ axes are the [100], [010] and [001] directions. The cubic anisotropy gives a weak angular dependence on the scale of several percent. The valance barrier height has been taken equal to $140 \mathrm{meV}$. The electron gas remains degenerate over the temperature range we considered $(<100$ $\mathrm{K})$. The Rabi frequencies $\Omega_{\mp, \mathrm{HH} 0}$ for the transition between $\left|J_{z}= \pm 3 / 2, l_{v}=0\right\rangle$ and $\left|S_{z}= \pm 1 / 2, l_{c}=0\right\rangle$ subbands are used to index the laser strength, $\Omega \equiv$ $\left(\left|\Omega_{+, \mathrm{HH} O}\right|^{2}+\left|\Omega_{-, \mathrm{HH}}\right|^{2}\right)^{1 / 2}$. For the Coulomb interaction, we follow the treatment of [23].

Fig 1(a) shows the band dispersions with and without dressing by $\sigma+$ polarized light with frequency tuned in resonance with the bandgap $E_{g} \equiv \varepsilon_{c}(0)-\varepsilon_{\mathrm{HH} 0}(0)$ (note that $\omega_{\text {edge }}$ is $\sim 10 \mathrm{meV}$ above $E_{g}$ ). The states near the $\Gamma$ point are strongly affected and the top dressed band acquires $k$-space Berry curvature with an $s$-like distribu- tion [Fig!1(b)]. The negative Berry curvature distribution for small $k$ is compensated by positive curvature for large $k$, so the Chern number of the top dressed band is still 0 . Nonetheless, there is a nonzero clean contribution to the carrier Hall conductance in each subspace $\mathcal{H}_{ \pm}$ $: \sigma_{ \pm}^{\mathrm{cl}}=e^{2} / \hbar \sum_{\mathbf{k}}(1-f) F_{z}^{c}(\mathbf{k})$, where $f$ gives the Fermi distribution of particles in the top dressed band. Since circularly polarized light breaks time reversal symmetry, there is a net charge Hall conductance $\sigma_{\mathrm{H}} \equiv \sigma_{+}+\sigma_{-}$with the magnitude controlled by the strength and frequency detuning of the laser [Fig. 1 (c)].

In a realistic crystal, we need to consider disorder effects. In a heavily modulation doped QW, the dominant cause of disorder is from dopants embedded in the barrier layers with smooth potentials in the QW layer. Electrons acquire an anomalous coordinate shift with magnitude proportional to the Berry curvature $F_{z}^{c}(\mathbf{k})$ when they scatter off a smooth impurity potential [24]. This leads to a side-jump contribution to the Hall conductance, $\sigma_{ \pm}^{\mathrm{sj}}=-e^{2} / \hbar \sum_{\mathbf{k}} F_{z}^{c}(\mathbf{k}) \frac{\partial f}{\partial \tilde{\varepsilon}_{c}} \frac{\partial \tilde{\varepsilon}_{c}}{\partial \mathbf{k}} \cdot \mathbf{k}$, independent of the scattering rate [10]. The admixture of valance band component also causes skew-scattering of conduction electrons by impurities [9], but its contribution to the Hall conductance is inversely proportional to the scattering rate and therefore is suppressed in this heavily doped case 25]. The total Hall conductance is then $\sigma^{\text {tot }} \equiv \sigma^{\text {cl }}+\sigma^{\text {sj }}$. Since $F_{z}^{c}(\mathbf{k})$ has strong $k$-dependence which changes with the light intensity, $\sigma^{\mathrm{sj}}$ has a more drastic change as a function of $\Omega$ and $\sigma^{\text {tot }}$ can even change sign [Fig 1 (c)].

With linearly polarized light, the $k$-space Berry curvature in the top dressed band has a $p$-like distribution [Fig 2 (b)]. The carrier Hall conductances in the two subspaces $\mathcal{H}_{ \pm}$have equal magnitude but opposite sign, $\sigma_{+}=-\sigma_{-}$, so the net charge Hall conductance vanishes. We define a pseudo-spin Hall conductance $\sigma_{s}=\hbar /(2 e)\left(\sigma_{+}-\sigma_{-}\right)$and its dependence on the laser strength and frequency detuning is studied in Fig 2 (c). We note that with circular polarized light, the charge Hall current typically has a majority contribution from one of the subspaces, so that there is a finite pseudo-spin Hall conductance as well [Fig[1(c)].

The sharpness of the spatial variation of the laser intensity is bounded by the optical wavelength $\lambda \sim \mu \mathrm{m}$ which is smooth enough to guarantee adiabatic evolution between the dressed $\tilde{b}_{c, \mathbf{k}}^{\dagger}\left|\Phi_{0}\right\rangle$ and undressed $b_{c, \mathbf{k}}^{\dagger}\left|\Phi_{0}\right\rangle$ at the light irradiation edges. The difference in the carrier Hall conductance $\sigma_{ \pm}$inside and outside the irradiation area leads to carrier accumulations $\Delta n_{ \pm}$at the edges in each subspace $\mathcal{H}_{ \pm}$, determined by the drift-diffusion equations: $\frac{\partial}{\partial t} \Delta n_{ \pm}-\frac{1}{e} \frac{\partial}{\partial y} \sigma_{ \pm} E_{x}-D \frac{\partial^{2}}{\partial y^{2}} \Delta n_{ \pm}=-\frac{\Delta n_{ \pm}-\Delta n_{\mp}}{\tau_{s}}$, where $\tau_{s}$ is the spin relaxation time, $L_{s} \equiv \sqrt{D \tau_{s}}$ the spin diffusion length, and momentum relaxation is assumed orders faster than $\tau_{s}$. For a sufficiently large electron density $n \gg \Delta n_{ \pm}$, the carrier accumulations are all in the top dressed bands. We define the pseudo-spin den- 


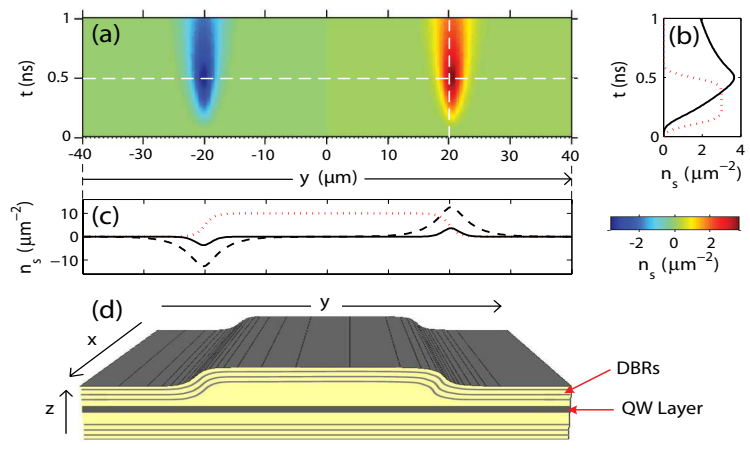

FIG. 3: (Color online) (a) Spatial and time dependence of spin accumulation $n_{s}$ driven by a DC electric field in the $x$ direction $(1 \mathrm{mV} / \mu \mathrm{m})$. The $\mathrm{SHE}$ is activated by a $\mathrm{X}$-polarized laser pulse with central frequency $\omega_{0}-E_{g}=13 \mathrm{meV}$. The temporal and spatial profile of the pulse is shown by the dotted curves in (b) and (c), with the peak strength at $y=0$ being $\Omega=5 \mathrm{meV}$. The system is assumed to be translationally invariant in $x$ direction in the region of interest. (b) Time dependence of the spin density (solid curve) at $y=20 \mu \mathrm{m}$. (c) Solid curve denotes spatial dependence of spin density at $t=0.5 \mathrm{~ns}$. The dashed curve denotes the steady state spin accumulation driven by a CW laser with the same spatial profile and $\Omega(y=0)=5 \mathrm{meV}$. (d) Schematic illustration of a QW embedded in an optical cavity [26]. The lowest cavity mode has spatial profile shown by the dotted curve in (c).

sity $n_{s}=\Delta n_{+}-\Delta n_{-}[16]$. Outside the irradiation area or after the light is adiabatically switched off, $n_{s}$ just gives the pure spin accumulation of the conduction band electrons. In Fig 3(a-c), we show $n_{s}$ generated by a laser pulse with duration $\sim 0.5 \mathrm{~ns}$ and a $\mathrm{CW}$ laser, assuming $\tau_{s}=5 \mathrm{~ns}[21]$ and $L_{s}=4 \mu \mathrm{m}$ [16].

In summary, we have shown that a sizable AHE and SHE due to dressed band topology can be optically activated in n-doped paramagnetic semiconductors. The absence of spin-orbit interactions when the light is switched off enables long spin lifetimes. With a reasonable value of the laser Rabi frequency $(\sim \mathrm{meV})$, optically activated topological spin Hall transport can be 3 orders stronger than that from skew scattering 16]. The laser excitation is operated in the reactive regime where the system excitation is predominantly virtual. Spontaneous emission of a photon at frequency $\omega_{0}-\tilde{\varepsilon}_{c}(\mathbf{k})+\tilde{\varepsilon}_{v}(\mathbf{k})$ (typically $>10$ $\mathrm{meV}$ below the laser resonance) can bring $\tilde{b}_{c,-\mathbf{k}}^{\dagger}\left|\Phi_{0}\right\rangle$ to $\tilde{b}_{v,-\mathbf{k}}^{\dagger}\left|\Phi_{0}\right\rangle[5]$. Thus, in free space, the system can have weak but finite absorption of energy from the light. For laser pulses with duration shorter than the spontaneous emission time $(\sim \mathrm{ns})$, the energy absorption would be small [5]. Embedding the QW in an optical cavity with a moderate $Q \sim 100$ [see Fig. 3(d)] can eliminate this unwanted dissipation. The cavity photon modes are lower bounded in frequency, and the spectrum edge, by design, is at the frequency of interest which allows in-couple of the laser and forbids out-couple at the spontaneous emission frequency. Cavity enhancement also allows a large Rabi frequency $\Omega$ to be achieved with a relatively weak external laser field.

We acknowledge useful discussions with X. F. Song, D. Xiao, X. Li and C. K. Shih. The work was supported by NSF, DOE and the Welch Foundation.

* Electronic address: wangyao@physics.utexas.edu

[1] C. Piermarocchi et al., Phys. Rev. Lett. 89, 167402 (2002); J. Fernandez-Rossier et al., ibid. 93, 127201 (2004).

[2] S. D. Ganichev et al., Nature 417, 153 (2002).

[3] M. J. Stevens et al., Phys. Rev. Lett. 90, 136603 (2003).

[4] J. Li et al., Appl. Phys. Lett. 88, 162105 (2006); X. Dai and F. C. Zhang, cond-mat/0605159 (2006).

[5] C. Comte and G. Mahler, Phys. Rev. B 34, 7164 (1986); S. Schmitt-Rink, D. S. Chemla, and H. Haug, ibid. 37, 941 (1988); S. Glutsch and R. Zimmermann, ibid. 45, 5857 (1992).

[6] D. J. Thouless et al., Phys. Rev. Lett. 49, 405 (1982).

[7] M.-C. Chang and Q. Niu, Phys. Rev. B 53, 7010 (1996).

[8] R. Karplus and J. M. Luttinger, Phys. Rev. 95, 1154 (1954).

[9] J. Smit, Physica 21, 877 (1955); ibid. 24, 39 (1958).

[10] L. Berger, Phys. Rev. B 2, 4559 (1970).

[11] T. Jungwirth, Q. Niu, and A. H. MacDonald, Phys. Rev. Lett. 88, 207208 (2002).

[12] Z. Fang et al., Science 302, 92 (2003).

[13] Y. Yao et al., Phys. Rev. Lett. 92, 037204 (2004).

[14] S. Onoda, N. Sugimoto, and N. Nagaosa, Phys. Rev. Lett. 97, 126602 (2006).

[15] S. Murakami, N. Nagaosa, and S.-C. Zhang, Science 301, 1348 (2003); J. Sinova et al., Phys. Rev. Lett. 92, 126603 (2004).

[16] Y. K. Kato et al., Science 306, 1910 (2004).

[17] J. Wunderlich et al., Phys. Rev. Lett. 94, 047204 (2005).

[18] J. Shi et al., Phys. Rev. Lett. 96, 076604 (2006).

[19] Y. A. Bychkov and E. I. Rashba, J. Phys. C: Solid State Phys. 17, 6039 (1984).

[20] B. A. Bernevig, T. L. Hughes, and S.-C. Zhang, Science 314, 1757 (2006); E. M. Hankiewicz, G. Vignale, and M. E. Flatté, Phys. Rev. Lett. 97, 266601 (2006).

[21] Y. Ohno et al., Phys. Rev. Lett. 83, 4196 (1999).

[22] D. A. Broido and L. J. Sham, Phys. Rev. B 31, 888 (1985).

[23] J. P. Eisenstein, in Nanophysics: Coherence and Transport, edited by H. Bouchiat et al. (Elsevier Science, 2005).

[24] N. A. Sinitsyn et al., Phys. Rev. B 72, 045346 (2005).

[25] Skew-scattering contribution can also be seperated from intrinsic and side-jump contributions by their different correlation with the longitudinal resistivity. See, C. Zeng et al., Phys. Rev. Lett. 96, 037204 (2006).

[26] A. Muller et al., Opt. Lett. 31, 528 (2006). 